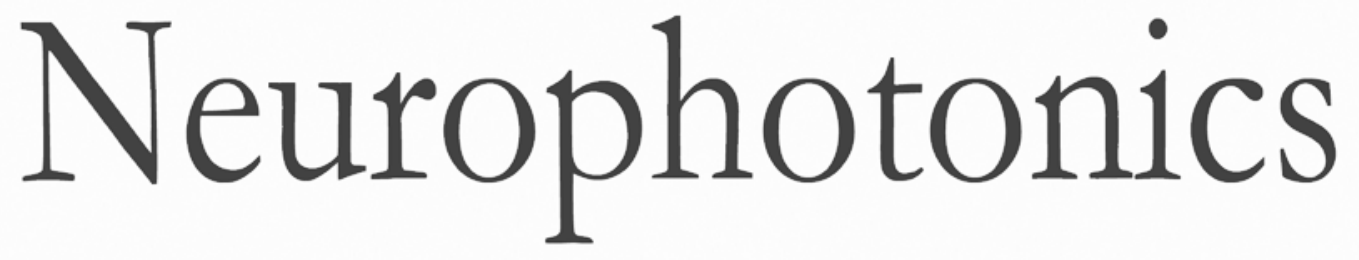

\title{
False positives and false negatives in functional near-infrared spectroscopy: issues, challenges, and the way forward
}

Ilias Tachtsidis

Felix Scholkmann 


\title{
False positives and false negatives in functional near-infrared spectroscopy: issues, challenges, and the way forward
}

\author{
Ilias Tachtsidis ${ }^{a}$ and Felix Scholkmann ${ }^{b, *}$ \\ aUniversity College London, Department of Medical Physics and Biomedical Engineering, Gower Street, Malet Place Engineering Building, \\ WC1E 6BT, London, United Kingdom \\ bUniversity Hospital Zurich, University of Zurich, Department of Neonatology, Biomedical Optics Research Laboratory, Frauenklinikstr. 10, \\ 8091 Zurich, Switzerland
}

\begin{abstract}
We highlight a significant problem that needs to be considered and addressed when performing functional near-infrared spectroscopy (fNIRS) studies, namely the possibility of inadvertently measuring fNIRS hemodynamic responses that are not due to neurovascular coupling. These can be misinterpreted as brain activity, i.e., "false positives" (errors caused by wrongly assigning a detected hemodynamic response to functional brain activity), or mask brain activity, i.e., "false negatives" (errors caused by wrongly assigning a not observed hemodynamic response in the presence of functional brain activity). Here, we summarize the possible physiological origins of these issues and suggest ways to avoid and remove them. ( The Authors. Published by SPIE under a Creative Commons Attribution 3.0 Unported License. Distribution or reproduction of this work in whole or in part requires full attribution of the original publication, including its DOI. [DOI: 10.1117/1.NPh.3.3.031405]
\end{abstract}

Keywords functional near-infrared spectroscopy; hemodynamic response.

Paper 15058NSSR received Dec. 16, 2015; accepted for publication Feb. 5, 2016; published online Mar. 9, 2016; corrected Mar. 15, 2016.

\section{Changes in Functional Near-Infrared Spectroscopy Signals Represent Neuronal Activity-But Not Always}

Functional near-infrared spectroscopy (fNIRS) is an increasingly used method in neuroscience research. It is a hemodynamic-based functional brain imaging technique, like functional magnetic resonance imaging (fMRI), relying on the detection of neurovascular coupling to infer changes in neuronal activity. Neurovascular coupling refers to the fact that changes in local neural activity are correlated with changes in local cerebral blood flow (CBF). The magnitude and spatial location of $\mathrm{CBF}$ changes are tightly linked to changes in neuronal activity. ${ }^{1,2}$ Neuronal excitation causes changes in oxidative metabolism (neurometabolic coupling) which is linked to neurovascular coupling; blood vessel dilation causes an increase in CBF and cerebral blood volume that overcompensates the metabolic demand. This oversupply of oxygenated blood (functional hyperemia), the cause of which is still a matter of debate, ${ }^{3}$ causes the fNIRS measurement of oxygenated hemoglobin $\left(\left[\mathrm{O}_{2} \mathrm{Hb}\right]\right)$ to increase, the deoxygenated hemoglobin $([\mathrm{HHb}])$ to decrease, and the total hemoglobin $\left([\mathrm{tHb}]=\left[\mathrm{O}_{2} \mathrm{Hb}\right]+[\mathrm{HHb}]\right)$ to increase. Although this physiological phenomenon is well known and expected, often it is not completely presented in publications (authors tend to focus only on the $\left[\mathrm{O}_{2} \mathrm{Hb}\right]$ response). In addition, there is a possibility that this pattern of $\left[\mathrm{O}_{2} \mathrm{Hb}\right],[\mathrm{HHb}]$, and $[\mathrm{tHb}]$ changes may not uniquely be caused by neurovascular coupling.

The aims of this opinion article are (i) to define and highlight the issue of false positives and negatives in fNIRS, (ii) to describe some of the challenges in recognizing these, and finally (iii) to suggest a way forward in resolving this issue. While

*Address all correspondence to: Felix Scholkmann, E-mail: Felix Scholkmann@usz.ch recently a substantial number of research and review articles (including ours) have discussed this issue; here, we will attempt to offer a concise and summarized view of the topic that will also represent our opinion. (In this opinion article, we explicitly assume that fNIRS instrumentation can accurately monitor and resolve the concentration changes in $\left[\mathrm{O}_{2} \mathrm{Hb}\right]$ and $[\mathrm{HHb}]$. The technical specifications and configuration of the fNIRS instrumentation (e.g., type and number of light sources and of photodetectors) can dictate the performance and accuracy of the device in monitoring $\left[\mathrm{O}_{2} \mathrm{Hb}\right]$ and $[\mathrm{HHb}]$. One aspect, in particular, is the number and choice of wavelengths used for resolving $\left[\mathrm{O}_{2} \mathrm{Hb}\right]$ and $[\mathrm{HHb}]$ as it has been reported that certain wavelength combinations can result in "crosstalk" between the chromophores. By crosstalk we mean to describe the phenomena that a genuine change in one chromophore concentration is also inducing a spurious measured concentration change in another; for a more detailed discussion of this issue see section "Selection of optimum wavelengths" in the recent review article by Scholkmann et al. ${ }^{4}$ and the recent study by Arifler et al. ${ }^{5}$ It is considered good practice for authors to report the wavelengths of their fNIRS instrument used in their study).

\section{What Are False Positives and False Negatives in Functional Near-Infrared Spectroscopy?}

We use the terms "false positives" or "false negatives" to state that during a functional experiment a change in the fNIRS signals $\left(\left[\mathrm{O}_{2} \mathrm{Hb}\right],[\mathrm{HHb}]\right.$, and $\left.[\mathrm{tHb}]\right)$ over a measurement channel that overlays a certain area of the cortex might not be due to the effect of neurovascular coupling, but could also be due to changes in (i) intracerebral hemodynamics caused by taskrelated systemic activity and/or (ii) extracerebral hemodynamics. These task-related systemic changes may mimic the 
presence of a neuronally induced hemodynamic response (false positives), or can attenuate (mask) the neuronally induced hemodynamic response (false negatives).

The presence of false positives and false negatives is not a problem exclusively for fNIRS, but is an issue for all hemodynamic-based functional brain imaging techniques including fMRI. However, the sensitivity of fNIRS to hemodynamic and oxygenation changes in the extracerebral (superficial) compartment is a particular confounding factor of this technique.

fNIRS is increasingly being used to investigate complicated cognitive functions, some of which can produce significant changes in systemic variables, which lead to non-neuronal driven changes in hemodynamics/oxygenation happening in the cerebral and extracerebral compartment mainly associated with changes in heart rate (HR), blood pressure, breathing rate, $\mathrm{CO}_{2}$ concentration in the blood, and autonomic nervous system (ANS) activity.

The presence of these systemic effects, and in an extension their consequences for fNIRS measurements, largely depends on the functional protocol (e.g., passive versus active tasks) and the individual subject studied (e.g., two people can react very differently to the same stressor).

\section{What Factors Cause False Positives and False Negatives in Functional Near-Infrared Spectroscopy?}

As described recently in detail, ${ }^{4}$ each fNIRS signal comprises different components that can be classified according to their (i) source (cerebral versus extracerebral), (ii) stimulus/task relation (evoked versus nonevoked), and (iii) physiological cause (neuronal versus systemic). The monitoring of the hemodynamic response due to neurovascular coupling that fNIRS aims to detect is only one of these components (i.e., the component neuronal/task-evoked/cerebral), while all the other components are the physiological noise that act as confounders in fNIRS studies (see Fig. 1).

Task-evoked changes in respiration and thus partial pressure of end-tidal carbon dioxide $\left(\mathrm{P}_{\mathrm{ET}} \mathrm{CO}_{2}\right)$, a reliable and accurate estimate of the partial pressure of carbon dioxide in the arterial blood $\left(\mathrm{PaCO}_{2}\right)$, was demonstrated in multimodal fNIRS studies (i.e., fNIRS studies including the measurements of systemic physiological changes using additional monitoring devices) investigating different tasks, e.g., speaking (maximal effect: $\left.\Delta \mathrm{PaCO}_{2} \approx-9 \mathrm{~mm} \mathrm{Hg}\right){ }^{6}$ mental arithmetic $\left(\Delta \mathrm{PaCO}_{2} \approx 0.5\right.$ to $3 \mathrm{~mm} \mathrm{Hg}$ ), ${ }^{6,7}$ and inner speech (i.e., speaking without vocalization, $\left.\Delta \mathrm{PaCO}_{2} \approx-0.5 \mathrm{~mm} \mathrm{Hg}\right){ }^{8}$

Task-evoked change in mean arterial blood pressure ( $\triangle \mathrm{MAP})$ has been demonstrated for a number of different experimental fNIRS protocols, e.g., arm-raising $(\Delta \mathrm{MAP} \approx 6 \mathrm{~mm} \mathrm{Hg}){ }^{9}$ visual stimulation $(\triangle \mathrm{MAP} \approx 2 \mathrm{~mm} \mathrm{Hg}),{ }^{10}$ anagram solving $\left(\Delta \mathrm{MAP} \approx 3\right.$ to $5 \mathrm{~mm} \mathrm{Hg},{ }^{11,12} 6$ to $\left.7 \mathrm{~mm} \mathrm{Hg}\right),{ }^{13}$ or hypercapnia $(\triangle \mathrm{MAP} \approx 4 \mathrm{~mm} \mathrm{Hg}),{ }^{14}$ hypocapnia $\left(\Delta \mathrm{MAP} \approx 4.5 \mathrm{~mm} \mathrm{Hg},{ }^{14}\right.$ $\Delta \mathrm{MAP} \approx-10 \mathrm{~mm} \mathrm{Hg}),{ }^{15}$ hypoxemia $(\Delta \mathrm{MAP} \approx 2 \mathrm{~mm} \mathrm{Hg}),{ }^{15}$ and hyperoxia $(\triangle \mathrm{MAP} \approx 2 \mathrm{~mm} \mathrm{Hg}) .{ }^{15}$

Since MAP is a function of cardiac output (CO), systemic vascular resistance (SVR), and central venous pressure (CVP) $(\mathrm{MAP}=\mathrm{CO} \times \mathrm{SVR}+\mathrm{CVP})$, and since $\mathrm{CO}$ is the product of $\mathrm{HR}$ and stroke volume $(\mathrm{SV})(\mathrm{CO}=\mathrm{HR} \times \mathrm{SV})$, task-evoked changes in HR and SVR will also have an effect on MAP.

\section{Sources of the hemodynamic response measured with fNIRS}

(a) Assumption

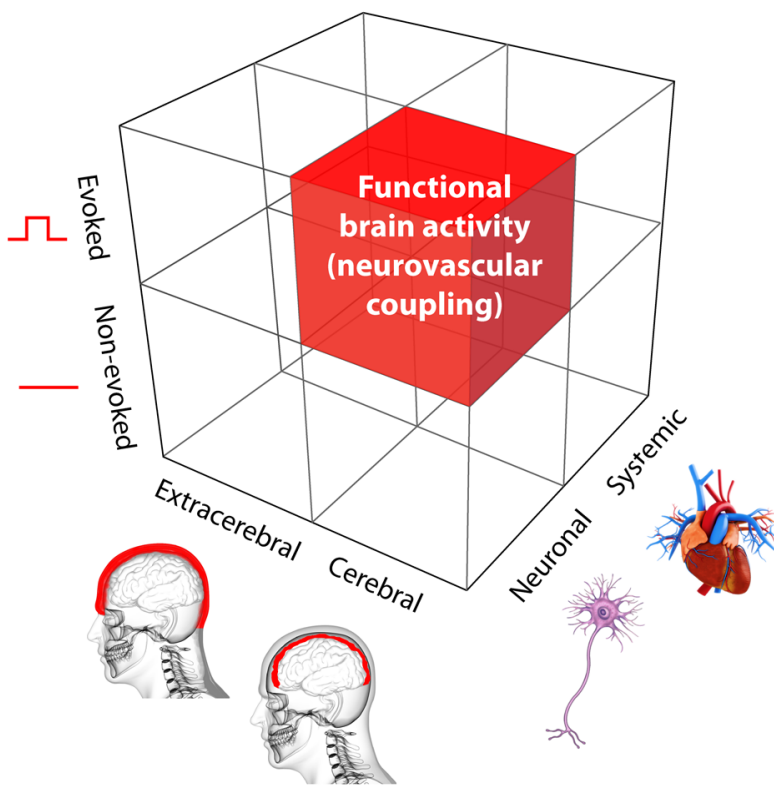

(b) Reality

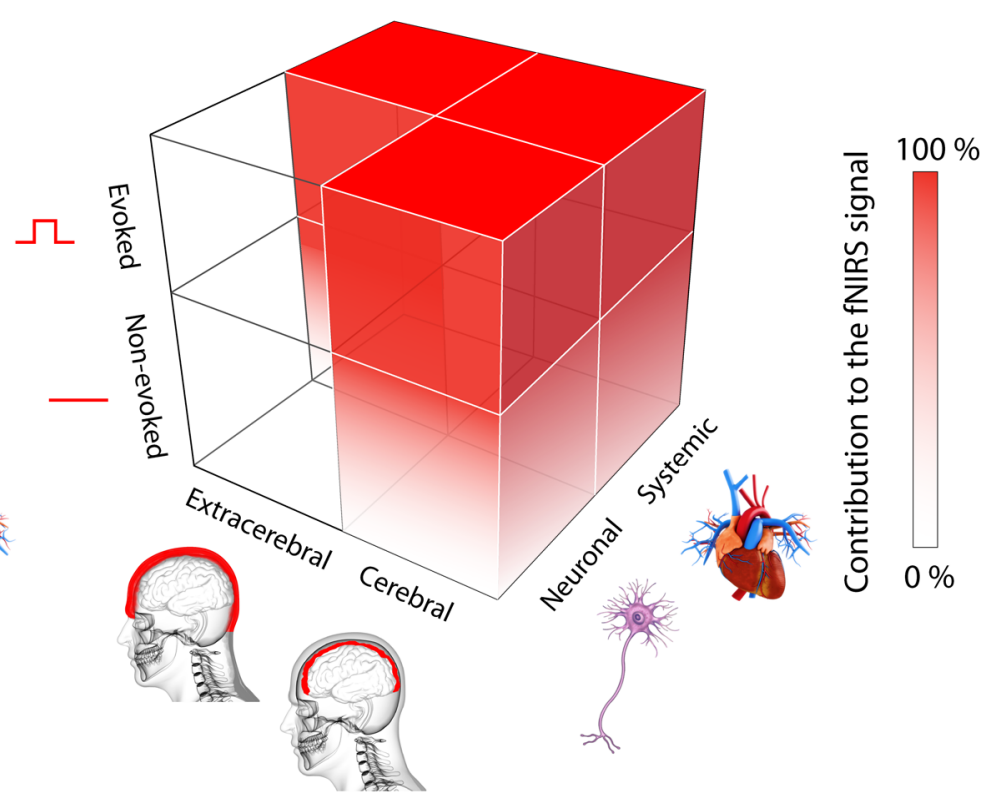

Fig. 1 Visualization of the six components of the fNIRS signals $\left(\left[\mathrm{O}_{2} \mathrm{Hb}\right],[\mathrm{HHb}],[\mathrm{tHb}]\right)$. (a) An erroneous assumption is that the fNIRS signal represents only changes associated with functional brain activity due to neurovascular coupling. (b) In reality, the fNIRS signals comprise six components so that five components are potentially confounders in every fNIRS study. The contribution of the components to the fNIRS signal is visualized by color-coding (red: $100 \%$, white: $0 \%$ ). The nonevoked/cerebral/neuronal and nonevoked/cerebral/systemic components can contribute to the fNIRS signal to a significant degree; however, the evoked changes can be generally stronger in an experimental paradigm assessing functional, task-related, brain-activity. 
A task-evoked change in the activity of the ANS is another factor potentially able to influence fNIRS signals since the ANS has a direct influence on CO, SVR, and in particular on blood flow (BF) in the extracerebral layer. All three of these physiological variables (i.e., respiration leading to $\mathrm{CO}_{2}$ changes, blood pressure, and ANS activity) affect the BF both in the cerebral compartment and in the extracerebral compartment (i.e., the scalp BF, ScBF). Changes in either the CBF or ScBF may have an impact on the recorded fNIRS signals.

The coupling strength between changes in blood pressure, $\mathrm{CO}_{2}$, or ANS activity with BF changes depends at least on two factors: (i) the type of compartment (cerebral versus extracerebral) and (ii) the speed of change (slow versus fast, i.e., minutes range versus seconds range). Table 1 shows the coupling strength values based on the present physiological knowledge. It is interesting to notice that the coupling is generally quite strong in all cases except between slow changes in blood pressure and $\mathrm{CBF}$, where the coupling is limited by dynamic cerebral autoregulation. Note, however, that the efficiency of cerebral autoregulation has also been controversially discussed in the recent literature, with doubt cast on the shape and boundaries of the CBF-transfer function as traditionally described. ${ }^{16}$

Extracerebral confounders are an issue because the diffusely reflected light in fNIRS is attenuated by a significant degree in the extracerebral tissue layer of the head and not only in the cerebral compartment. ${ }^{18-21}$ The penetration depth $(D)$ of light emitted in the head tissue layers is substantially less than half the source-detector separation (SDS) as predicted by the diffusion approximation ${ }^{22}$ (i.e., $D<0.5$ SDS). Changes in hemodynamics and oxygenation in the extracerebral layer will have a strong impact on the fNIRS signals. This issue has been known in principle for many years (e.g., Refs. 2327) but the real significance becoming increasingly apparent with a growing number of studies showing that fNIRS is affected by these extracerebral changes and also that the extracerebral hemodynamic changes are spatially heterogeneous. ${ }^{28-31}$

\section{How Can We Avoid and/or Remove False Positives and Negatives in Functional Near-Infrared Spectroscopy Studies?}

False positives and negatives can be avoided, minimized, and/or dealt with by adopting one or more of the following approaches (listed in no particular order of significance): (i) careful experimental design (e.g., avoiding tasks with strong systemic activation, avoiding additional stress and discomfort for the subject), (ii) depth-resolved fNIRS techniques [e.g., multidistance (MD) measurements], (iii) appropriate statistical and/or signal processing methods [e.g., independent-component analysis (ICA), principal component analysis (PCA), adaptive filtering, analysis with a general linear model (GLM) with systemic regressors], (iv) multimodal monitoring (e.g., measurement of local ScBF or measurements of signals from the systemic physiology).

(i) Designing the fNIRS protocol: As in any neuroscience study, careful consideration should be taken in selecting the trial design, defining the experimental tasks, designing the contrasts, and choosing the repetition and duration times. The fundamental aspect to consider when designing an fNIRS protocol is that brain activation must be assessed relative to a control condition or baseline. The functional protocol needs to have the best possible baseline condition to subtract out "spurious" hemodynamic/oxygenation responses from the experimental task. fNIRS protocols must thus create a high contrast between either the experimental condition and the baseline condition or between two experimental conditions.

(ii) fNIRS instrumentation: Concerning fNIRS hardware optimization, there are different possibilities to reduce the influence of physiological confounders in the recorded signals. Time-domain fNIRS devices offer the possibility to distinguish between measured changes in the cerebral or extracerebral layer automatically due to the measured temporal photon point-spread function. New algorithms and methods to separate the contribution from the extracerebral and cerebral compartment were published recently, e.g., Refs. 32 and 33. For continuous-wave fNIRS (cwfNIRS) and frequency-domain fNIRS systems, MD measurements enable increased sensitivity to the cerebral compartment by calculating the slopes of the decrease in light intensity as a function of SDSs. The MD approach can be implemented via two main techniques "spatially resolved spectroscopy" and the "self-calibrating method" (for a more detailed description see the recently published review. ${ }^{4}$ ) Another possibility is to record with extra short channels. The signal from these short channels can then be used to regress out influences from the extracerebral layer. ${ }^{34}$ The optimal SDS for such short-channel regression was determined recently to be $8.4 \mathrm{~mm}$ for adults and $2.15 \mathrm{~mm}$ for a term-age infant. ${ }^{35}$ The number and location of short channels used should be guided by anatomical/ physiological constraints, as discussed recently by Zhang et al. ${ }^{28}$ Specific signal processing and data analysis methods to reduce physiological confounding include approaches that use information from the short channels or that are based on the different dynamics of $\left[\mathrm{O}_{2} \mathrm{Hb}\right]$

Table 1 Overview (according to our own opinion based on published literature and own experience) of the coupling strength between BF and systemic variables, i.e., blood pressure, $\mathrm{PaCO}_{2}$, and ANS activity. "*” indicates that at present the coupling strength is not known or controversially discussed. For a critical review of the coupling factors, see Refs. 16 and 17. Coupling between hemodynamic changes in the cerebral layer concern the CBF, whereas changes in the extracerebral compartment are linked to scalp BF.

\begin{tabular}{lcccccc} 
& \multicolumn{2}{c}{$\begin{array}{c}\text { Coupling strength between } \\
\text { MAP and BF }\end{array}$} & & \multicolumn{2}{c}{$\begin{array}{c}\text { Coupling strength between } \\
\mathrm{PaCO}_{2} \text { and } \mathrm{BF}\end{array}$} \\
\cline { 2 - 3 } Tissue compartment & Slow change & Fast change & & Slow change & Fast change & Coupling strength between ANS and BF \\
\hline Extracerebral $(\rightarrow \mathrm{ScBF})$ & High & High & & $*$ & $*$ & High \\
Cerebral $(\rightarrow \mathrm{CBF})$ & Low & High & High & High & Low \\
\hline
\end{tabular}


and $[\mathrm{HHb}]$. For a general overview of these methods, see the section "Signal analysis methods to extract the functional brain activity" in Ref. 4 as well as the review of Tak and Ye. ${ }^{36}$

Another aspect to consider concerns the optode attachment. It is generally assumed that a tight attachment of the optodes to the scalp can eliminate or minimize the extracerebral contamination by squeezing out the scalp blood. While this is certainly true and may help to reduce the influence of the hemodynamic changes in the extracerebral layer, it must also be noted that the high probe pressure on the head can result in discomfort and local pain which activates the ANS leading to an increased strength of the systemic confounders.

The probe pressure can also be varied and, in combination with an MD measurement, an approach recently developed by Baker et al. ${ }^{37}$ can be used to remove the hemodynamic influences from the extracerebral layer from the fNIRS signals.

(iii) Analysis tools and approaches: The analysis of all these signals within a single framework is challenging. One possibility is to employ a GLM where the physiological signals are additional regressors, as demonstrated already in a few studies. $^{29,30,38}$ In addition, the simultaneous use of $\left[\mathrm{O}_{2} \mathrm{Hb}\right]$ and $[\mathrm{HHb}]$ in the same model may enhance the correct detection of the hemodynamic response and improve the statistics. Another useful approach is to use ICA in combination with MD measurements as recently shown. ${ }^{39-42}$ Combining the ICA approach with systemic measurements is promising. ${ }^{43}$ In a recent study, Kirilina et al. ${ }^{30}$ proposed a denoising algorithm that uses regressors derived from the time dependency between the fNIRS and systemic signals. And the usefulness to use PCA in order to separate the global and local components in fNIRS signals was shown recently. ${ }^{44}$

Since the strength of task-evoked systemic physiological changes depends significantly on the specific psychophysiological state of the subject, single-level data analysis might also be important to avoid false positives and negatives. A combination of single-level and group-level analyses may be the optimal approach.

(iv) Multimodal monitoring: The fNIRS experimental environment allows easy interfacing with additional instruments that can monitor systemic variables during the functional experiment. These can be devices enabling the measurement of MAP, respiration, $\mathrm{P}_{\mathrm{ET}} \mathrm{CO}_{2}, \mathrm{HR}$, skin conductance, and $\mathrm{ScBF}$. These measurements may then be used to identify individuals that exhibit large task-related systemic changes that can interfere with the brain functional response and thus exclude them from further analysis. These measurements can also be employed to validate the systemic similarities between the baseline and experimental task conditions. Importantly, these measurements can be used in statistical and signal processing methods as regressors and independent components to account for the non-neuronal-related functional response.

Although we have focused above on fNIRS monitoring during functional paradigms, there are additional issues to be considered when there is no functional contrast, i.e., in a study assessing the resting-state brain activity. Both neuronal and non-neuronal hemodynamic generating factors are present during the resting-state and should be considered when designing the experimental protocol [e.g., possible environmental noise, change in resting-state activity due to increased drowsiness during the experiment, increased subject stress due to the fNIRS optode(s) attachment which can cause unpleasant sensations and thus induce a change in ANS activity]. When conducting resting-state fNIRS studies one should be aware of the fact that more and more studies report the influence of systemic hemodynamic changes on resting-state fNIRS and fMRI signals. For example, recently the findings of Tong et al. ${ }^{45}$ suggested, based on an fMRI study, that the resting-state "may reflect, to some extent, vascular anatomy associated with systemic fluctuations, rather than neuronal connectivity." Also, Zhu et al. ${ }^{46}$ recently concluded from their resting-state (rs) fMRI findings that they "raise a critical question of whether a large portion of rs-fMRI signals can be attributed to the vascular effects produced from upstream changes in cerebral hemodynamics." Since fNIRS signals are additionally affected by changes in $\mathrm{ScBF}$, careful interpretation of resting-state fNIRS signals is warranted. For resting-state fNIRS studies, it thus makes sense to name the measured connectivity accordingly, i.e., "vascular connectivity" 47 or "sum of intracerebral and extracerebral hemodynamic functional connectivity." 48

\section{Way Forward}

There are many factors that should be considered in order to improve future fNIRS studies. What needs to be recognized immediately is the imperativeness of considering the time dynamics of both $\left[\mathrm{O}_{2} \mathrm{Hb}\right]$ and $[\mathrm{HHb}]$ signals in fNIRS data analysis, and their interrelationship before formulating any neuroscientific conclusions. Reporting both $\left[\mathrm{O}_{2} \mathrm{Hb}\right]$ and $[\mathrm{HHb}]$ changes, instead of only one of them, allows better physiological interpretation of the functional experimental results (e.g., when both $\left[\mathrm{O}_{2} \mathrm{Hb}\right]$ and $[\mathrm{HHb}]$ show a strong increase and/or when both $\left[\mathrm{O}_{2} \mathrm{Hb}\right]$ and $[\mathrm{HHb}]$ show a strong decrease during the task periods then the signals are probably confounded by systemic changes). However observing the typical pattern of the hemodynamic response during functional activation (i.e., increase in $\left[\mathrm{O}_{2} \mathrm{Hb}\right]$ and decrease in $[\mathrm{HHb}]$ ) is not sufficient for concluding that no systemic confounding effect took place. Synchronous measurements of systemic physiology, currently easily achievable with highly portable and relatively inexpensive instrumentation, can then also provide an accessible way to regress out systemic interference and identify individuals with strong task-related systemic changes.

Both $\left[\mathrm{O}_{2} \mathrm{Hb}\right]$ and $[\mathrm{HHb}]$ can be influenced by systemic physiological changes (occurring in the cerebral and/or the extracerebral compartment). However, the degree of the influence often varies and also depends on the origin and type of the systemic change. Kirilina et al. ${ }^{29}$ demonstrated that $\left[\mathrm{O}_{2} \mathrm{Hb}\right]$ signals are more strongly affected by global processes in both extracerebral and intracerebral compartments, and local scalp BF regulation, while [HHb] signals are less contaminated by extracerebral processes. The physiological reason for this may be the fact that arterioles are enervated to a larger degree by the fibers from the sympathetic nervous system than the venules; ${ }^{49,50}$ during activity of the ANS, the $\left[\mathrm{O}_{2} \mathrm{Hb}\right]$ signals seem to be more affected than the [HHb] signals-a conclusion also supported by recent findings of Haeussinger et al. ${ }^{51}$ In addition, it is well known that cardiac oscillations are more 
prominent in the $\left[\mathrm{O}_{2} \mathrm{Hb}\right]$ compared to the $[\mathrm{HHb}]$. Although there is evidence of the $[\mathrm{HHb}]$ signal to be less contaminated by systemic changes, this does not imply that the $[\mathrm{HHb}]$ signal is not confounded by systemic changes.

In the present state of fNIRS research, most studies are using cw-fNIRS systems that implement only measurements at a fixed $3 \mathrm{~cm}$ SDS. In this case, it is recommended that ScBF effects be corrected by either (i) adding additional short channels and using them to regress out the superficial hemodynamics (by using one of the short-channel regression methods developed so far, e.g., Refs. 39, 41, and 52-56), (ii) by calculating the mean signal over all channels and using this channel as the superficial regressor (e.g., Refs. 57 and 58), or (iii) by combining both of these approaches. ${ }^{59}$

More and more fNIRS instrumentation companies currently provide alternative and/or add-on solutions to allow the implementation of MD probe geometries with subsequent shortchannel regression frameworks. In the near future, such instrumentation advances will allow fNIRS measurements that better represent the neuronal, cerebral, and evoked responses.

\section{Acknowledgments}

We thank Professor Dr. Martin Wolf for helpful comments on an earlier draft of this paper, and Rachel Scholkmann and Dr. Matthew Caldwell for proofreading this paper. Dr. Ilias Tachtsidis is a Wellcome Trust senior fellow and acknowledges funding from this trust (104580/Z/14/Z).

\section{References}

1. A. Devor et al., "Neuronal basis of non-invasive functional imaging: from microscopic neurovascular dynamics to BOLD fMRI," $A d v$. Neurobiol. 4, 433-500 (2012).

2. R. B. Buxton, "Dynamic models of BOLD contrast," NeuroImage 62(2), 953-961 (2012).

3. C. Leithner and G. Roy, "The oxygen paradox of neurovascular coupling," J. Cereb. Blood Flow Metab. 34, 19-29 (2014).

4. F. Scholkmann et al., "A review on continuous wave functional nearinfrared spectroscopy and imaging instrumentation and methodology," Neurolmage 85(Pt 1), 6-27 (2014).

5. D. Arifler et al., "Optimal wavelength combinations for near-infrared spectroscopic monitoring of changes in brain tissue hemoglobin and cytochrome c oxidase concentrations," Biomed. Opt. Express 6(3), 933-947 (2015).

6. F. Scholkmann et al., "End-tidal $\mathrm{CO}_{2}$ : an important parameter for a correct interpretation in functional brain studies using speech tasks," NeuroImage 66, 71-79 (2013).

7. F. Scholkmann, M. Wolf, and U. Wolf, "The effect of inner speech on arterial $\mathrm{CO}_{2}$, cerebral hemodynamics and oxygenation-a functional NIRS study," Adv. Exp. Med. Biol. 789, 81-87 (2013).

8. F. Scholkmann et al., "Cerebral hemodynamic and oxygenation changes induced by inner and heard speech: a study combining functional nearinfrared spectroscopy and capnography," J. Biomed. Opt. 19(1), 017002 (2014).

9. L. Minati et al., "Intra- and extra-cranial effects of transient blood pressure changes on brain near-infrared spectroscopy (NIRS) measurements," J. Neurosci. Methods 197(2), 283-288 (2011).

10. L. Minati et al., "Emotional modulation of visual cortex activity: a functional near-infrared spectroscopy study," NeuroReport 20(15), 13441350 (2009).

11. I. Tachtsidis et al., "Investigation of frontal cortex, motor cortex and systemic haemodynamic changes during anagram solving," Adv. Exp. Med. Biol. 614, 21-28 (2008).

12. I. Tachtsidis et al., "Measurement of frontal lobe functional activation and related systemic effects: a near-infrared spectroscopy investigation," Adv. Exp. Med. Biol. 614, 397-403 (2008).
13. I. Tachtsidis et al., "False positives in functional near-infrared topography," Adv. Exp. Med. Biol. 645, 307-314 (2009).

14. J. M. Serrador et al., "MRI measures of middle cerebral artery diameter in conscious humans during simulated orthostasis," Stroke 31, 16721678 (2000).

15. M. M. Tisdall et al., "The effect on cerebral tissue oxygenation index of changes in the concentrations of inspired oxygen and end-tidal carbon dioxide in healthy adult volunteers," Anesth. Analg. 109(3), 906-913 (2009).

16. C. K. Willie et al., "Integrative regulation of human brain blood flow," J. Physiol. 592(Pt 5), 841-859 (2014).

17. P. N. Ainslie and P. Brassard, "Why is the neural control of cerebral autoregulation so controversial?," F1000Prime Rep. 6, 14 (2014).

18. S. Gunadi et al., "Spatial sensitivity and penetration depth of three cerebral oxygenation monitors," Biomed. Opt. Express 5(9), 2896-2912 (2014).

19. V. Toronov et al., "Near-infrared study of fluctuations in cerebral hemodynamics during rest and motor stimulation: temporal analysis and spatial mapping," Med. Phys. 27(4), 801-815 (2000).

20. G. E. Strangman, Q. Zhang, and Z. Li, "Scalp and skull influence on near infrared photon propagation in the Colin27 brain template," Neurolmage 85(Pt 1), 136-149 (2014).

21. P. G. Al-Rawi, P. Smielewski, and P. J. Kirkpatrick, "Evaluation of a near-infrared spectrometer (NIRO 300) for the detection of intracranial oxygenation changes in the adult head," Stroke 32(11), 2492-2500 (2001).

22. A. V. Patil et al., "Experimental investigation of NIRS spatial sensitivity," Biomed. Opt. Express 2(6), 1478-1493 (2011).

23. T. J. Germon et al., "Cerebral near infrared spectroscopy: emitter-detector separation must be increased," Br. J. Anaesth. 82(6), 831-837 (1999).

24. T. J. Germon et al., "Sensitivity of near infrared spectroscopy to cerebral and extra-cerebral oxygenation changes is determined by emitter-detector separation," J. Clin. Monit. Comput. 14(5), 353-360 (1998).

25. D. N. Harris, F. M. Cowans, and D. A. Wertheim, "NIRS in the temporal region-strong influence of external carotid artery," Adv. Exp. Med. Biol. 345, 825-828 (1994).

26. C. Mansouri et al., "Depth sensitivity analysis of functional near-infrared spectroscopy measurement using three-dimensional Monte Carlo modelling-based magnetic resonance imaging," Lasers Med. Sci. 25, 431-438 (2010).

27. J. Selb et al., "Sensitivity of near-infrared spectroscopy and diffuse correlation spectroscopy to brain hemodynamics: simulations and experimental findings during hypercapnia," Neurophotonics 1(1), 015005 (2014).

28. Y. Zhang et al., "Multiregional functional near-infrared spectroscopy reveals globally symmetrical and frequency-specific patterns of superficial interference," Biomed. Opt. Express 6(8), 2786-2802 (2015).

29. E. Kirilina et al., "The physiological origin of task-evoked systemic artefacts in functional near infrared spectroscopy," NeuroImage 61(1), 70-81 (2012).

30. E. Kirilina et al., "Identifying and quantifying main components of physiological noise in functional near infrared spectroscopy on the prefrontal cortex," Front. Hum. Neurosci. 7, 864 (2013).

31. T. Nasi et al., "Effect of task-related extracerebral circulation on diffuse optical tomography: experimental data and simulations on the forehead," Biomed. Opt. Express 4(3), 412-426 (2013).

32. A. Jelzow et al., "Separation of superficial and cerebral hemodynamics using a single distance time-domain NIRS measurement," Biomed. Opt. Express 5(5), 1465-1482 (2014).

33. J. Selb et al., "Comparison of a layered slab and an atlas head model for Monte Carlo fitting of time-domain near-infrared spectroscopy data of the adult head," J. Biomed. Opt. 19(1), 016010 (2014).

34. M. A. Yücel et al., "Short separation regression improves statistical significance and better localizes the hemodynamic response obtained by near-infrared spectroscopy for tasks with differing autonomic responses," Neurophotonics 2(3), 035005 (2015).

35. S. Brigadoi and R. J. Cooper, "How short is short? Optimum sourcedetector distance for short-separation channels in functional near-infrared spectroscopy," Neurophotonics 2(2), 025005 (2015).

36. S. Tak and J. C. Ye, "Statistical analysis of fNIRS data: a comprehensive review," Neurolmage 85(Pt 1), 72-91 (2014).

37. W. B. Baker et al., "Pressure modulation algorithm to separate cerebral hemodynamic signals from extracerebral artifacts," Neurophotonics 2(3), 035004 (2015). 
38. I. Tachtsidis et al., "Functional optical topography analysis using statistical parametric mapping (SPM) methodology with and without physiological confounds," Adv. Exp. Med. Biol. 662, 237-243 (2010).

39. T. Funane et al., "Quantitative evaluation of deep and shallow tissue layers' contribution to fNIRS signal using multi-distance optodes and independent component analysis," Neurolmage 85(Pt 1), 150165 (2014).

40. T. Funane et al., "Greater contribution of cerebral than extracerebral hemodynamics to near-infrared spectroscopy signals for functional activation and resting-state connectivity in infants," Neurophotonics 1(2), 025003 (2014).

41. T. Funane et al., "Concurrent fNIRS-fMRI measurement to validate a method for separating deep and shallow fNIRS signals by using multidistance optodes," Neurophotonics 2(1), 015003 (2015).

42. M. Kiguchi and T. Funane, "Algorithm for removing scalp signals from functional near-infrared spectroscopy signals in real time using multidistance optodes," J. Biomed. Opt. 19(11), 110505 (2014).

43. S. Patel et al., "Quantification of systemic interference in optical topography data during frontal lobe and motor cortex activation: an independent component analysis," Adv. Exp. Med. Biol. 701, 45-51 (2011).

44. X. Zhang, J. A. Noah, and J. Hirsch, "Separation of the global and local components in functional near-infrared spectroscopy signals using principal component spatial filtering," Neurophotonics 3(1), 015004 (2016).

45. Y. Tong et al., "Can apparent resting state connectivity arise from systemic fluctuations?," Front. Hum. Neurosci. 9, 285 (2015).

46. D. C. Zhu et al., "Vascular coupling in resting-state fMRI: evidence from multiple modalities," J. Cereb. Blood Flow Metab. 35(12), 1910-1920 (2015).

47. U. Wolf et al., "Correlation of functional and resting state connectivity of cerebral oxy-, deoxy-, and total hemoglobin concentration changes measured by near-infrared spectrophotometry," J. Biomed. Opt. 16(8), 087013 (2011).

48. L. Holper, F. Scholkmann, and E. Seifritz, "Time-frequency dynamics of the sum of intra-and extracerebral hemodynamic functional connectivity during resting-state and respiratory challenges assessed by multimodal functional near-infrared spectroscopy," NeuroImage 120, 481492 (2015).

49. K. Kogure et al., "Adrenergic control of cerebral blood flow and energy metabolism in the rat," Stroke 10(2), 179-184 (1979).

50. G. D. Hirst and F. R. Edwards, "Sympathetic neuroeffector transmission in arteries and arterioles," Physiol. Rev. 69(2), 546-604 (1989).
51. F. B. Haeussinger et al., "Reconstructing functional near-infrared spectroscopy (fNIRS) signals impaired by extra-cranial confounds: an easyto-use filter method," NeuroImage 95, 69-79 (2014).

52. R. B. Saager and A. J. Berger, "Direct characterization and removal of interfering absorption trends in two-layer turbid media," J. Opt. Soc. Am. A 22(9), 1874-1882 (2005).

53. R. B. Saager and A. J. Berger, "Measurement of layer-like hemodynamic trends in scalp and cortex: implications for physiological baseline suppression in functional near-infrared spectroscopy," J. Biomed. Opt. 13(3), 034017 (2008).

54. R. B. Saager, N. L. Telleri, and A. J. Berger, "Two-detector corrected near infrared spectroscopy (C-NIRS) detects hemodynamic activation responses more robustly than single-detector NIRS," NeuroImage 55(4), 1679-1685 (2011).

55. L. Gagnon et al., "Further improvement in reducing superficial contamination in NIRS using double short separation measurements," Neurolmage 85(Pt 1), 127-135 (2014).

56. F. Scarpa et al., "A reference-channel based methodology to improve estimation of event-related hemodynamic response from fNIRS measurements," NeuroImage 72, 106-119 (2013).

57. G. Pfurtscheller et al., "Focal frontal (de)oxyhemoglobin responses during simple arithmetic," Int. J. Psychophysiol. 76(3), 186-192 (2010).

58. G. Bauernfeind et al., "Separating heart and brain: on the reduction of physiological noise from multichannel functional near-infrared spectroscopy (fNIRS) signals," J. Neural Eng. 11(5), 056010 (2014).

59. S. B. Erdoğan, M. A. Yücel, and A. Akın, "Analysis of task-evoked systemic interference in fNIRS measurements: insights from fMRI," NeuroImage 87, 490-504 (2014).

Ilias Tachtsidis received his $\mathrm{PhD}$ at the University College London (UCL), UK, in 2005. He is a senior member of the Biomedical Optics Research Laboratory at UCL and a senior Wellcome Trust fellow. He works on the development and application of NIRS techniques to monitor the function of the brain both in health and disease, including traumatic brain injury, and neonatal encephalopathy.

Felix Scholkmann received his $\mathrm{PhD}$ at the University of Zurich, Switzerland, in 2014. A postdoc and research associate at the Biomedical Optics Research Laboratory of the University Hospital Zurich, his research focuses on biomedical signal processing, biomedical optics (development and application of NIRS), neuroscience, integrative physiology, and biophysics. 